\title{
Concurrent cavitary pulmonary tuberculosis and COVID-19 pneumonia with in vitro immune cell anergy
}

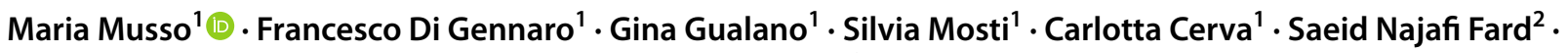 \\ Raffaella Libertone ${ }^{1} \cdot$ Virginia Di Bari $^{1} \cdot$ Massimo Cristofaro $^{3} \cdot$ Roberto Tonnarini $^{1}$. Concetta Castilletti ${ }^{4}$. \\ Delia Goletti ${ }^{2}$ Fabrizio Palmieri ${ }^{1}$
}

Received: 24 June 2020 / Accepted: 2 January 2021 / Published online: 17 January 2021

(c) Springer-Verlag GmbH, DE part of Springer Nature 2021

\begin{abstract}
Tuberculosis (TB) is top infectious disease killer caused by a single organism responsible for 1.5 million deaths in 2018 . Both COVID-19 and the pandemic response are risking to affect control measures for TB and continuity of essential services for people affected by this infection in western countries and even more in developing countries. Knowledge about concomitant pulmonary TB and COVID-19 is extremely limited. The double burden of these two diseases can have devastating effects. Here, we describe from both the clinical and the immunological point of view a case of a patient with in vitro immune cell anergy affected by bilateral cavitary pulmonary TB and subsequent COVID-19-associated pneumonia with a worst outcome. COVID-19 can be a precipitating factor in TB respiratory failure and, during ongoing SARS-COV-2 pandemic, clinicians must be aware of this possible co-infection in differential diagnosis of patients with active TB and new or worsening chest imaging.
\end{abstract}

Keywords COVID-19 $\cdot$ Tuberculosis $\cdot$ SARS-CoV-2 $\cdot$ IGRA test

\section{Background}

Experience with concomitant TB and COVID-19 is extremely limited. Both M. tuberculosis and SARS-CoV-2 affect primarily the lungs and interfere with host immunity [1]. Here, we describe a case of a patient with in vitro immune cell anergy affected by bilateral cavitary pulmonary TB and subsequent COVID-19-associated pneumonia with a worst outcome. The patient was also included in a cohort of 69 patients [2]; here, we present a deeper focus on clinical and moreover immunological features.

Maria Musso

maria.musso@inmi.it

1 Respiratory Infectious Diseases Unit, National Institute for Infectious Diseases “L. Spallanzani” IRCCS, Rome, Italy

2 Translational Research Unit, National Institute for Infectious Diseases “L. Spallanzani”, IRCCS, Rome, Italy

3 Diagnostic Imaging Unit, National Institute of Infectious Diseases “L. Spallanzani”, IRCCS, Rome, Italy

4 Laboratory of Virology, National Institute of Infectious Diseases “L. Spallanzani”, IRCCS, Rome, Italy

\section{Case presentation}

During the COVID-19 outbreak in Italy, a 45-year-old man of Moldovan origin presented himself to an Emergency Department of another hospital referring TB-like symptoms as cough, fatigue and weight loss since 3 months before. He has been always in good health, to note only an ethilism addiction in the clinical history. During hospitalization in the Emergency Department, a thorax CT scan showed multiple cavitary lesions on the right lung and an idropneumothorax with complete atelectasis of left lung (Fig. 1a). Acid-fast bacilli were present in the sputum and standard therapy with rifampicin, isoniazid, ethambutol and pyrazinamide was started. Qualitative real-time reverse-transcriptase-polymerase chain reaction (RT-PCR) for SARS-CoV-2 (Altona Diagnostics GmbH, Germany) on two nasopharyngeal swabs was negative. Arterial blood gas analysis showed mild impairment with arterial oxygen tension $\left(\mathrm{PaO}_{2}\right) 102 \mathrm{mmHg}$; fraction of inspired oxygen $\left(\mathrm{FiO}_{2}\right)$ was $31 \%$ with a $\mathrm{PaO} 2 / \mathrm{FiO}_{2}$ ratio of $329 \mathrm{mmHg}$. A chest tube drainage was positioned for idropneumothorax and after seven days, he was referred to $\mathrm{L}$. Spallanzani Institute in Rome with a diagnosis of pulmonary TB and idropneumothorax. 

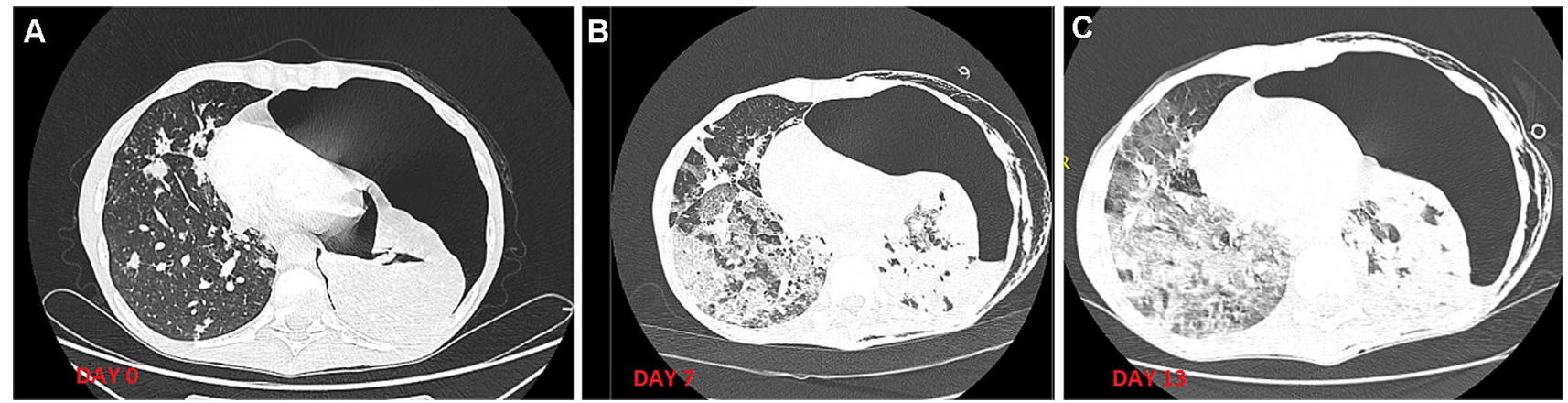

Fig. 1 Patient's chest CT at admission in the emergency department (a), after seven days (b) and after thirteen days (c) (6 days from the admission to "L. Spallanzani" Institute)

Physical examination at admission in our Institute showed cachexia (Body Mass Index: 19). Respiratory rate was normal (RR 12/min) but he presented respiratory failure. Arterial blood gas analysis revealed moderate impairment with $\mathrm{PaO}_{2} 67 \mathrm{mmHg}\left(\mathrm{FiO}_{2}\right.$ was $\left.35 \%\right)$ and $\mathrm{PaO} 2 / \mathrm{FiO}_{2}$ ratio of $191 \mathrm{mmHg}$. Tachycardia was present at 116 beats per minute with a blood pressure at $120 / 60 \mathrm{mmHg}$; body temperature was $36{ }^{\circ} \mathrm{C}$. No vesicular murmur was present at left lung auscultation. A significant subcutaneous emphysema involved the left chest. A high-resolution computed tomography (HRCT) of the chest was repeated (day 7 from admission to Emergency Department) and showed an incomplete re-expansion of the lung despite a well-positioned drainage and a new appearance of multiple patchy ground grass opacities with pattern "crazy paving" in the right lung (Fig. 1b).

Blood routine tests revealed liver function impairment with aspartate aminotransferase/alanine aminotransferase 87/130 U/L, albumin $2 \mathrm{mg} / \mathrm{dl}$, increased C-reactive protein (7.55 mg/dl) and lymphophenia (735 lymphocytes/mmc with a percentage of $11.1 \%$ ). HIV test was negative. Numerous acid-fast bacilli were present in the sputum; M. tuberculosis (Real-time PCR Anyplex, Seegene Inc. Republic of Korea) indicated sensitivity to rifampin, isoniazid, fluorochinolones and aminoglycosides. Anti-tubercular regimen was modified for liver function impairment according to the institutional protocol, including rifampicin, ethambutol, amikacin and moxifloxacin. At admission, he underwent again nasopharingeal swab with detection of SARS-CoV-2 (Altona Diagnostics GmbH, Germany). Hidroxicloroquine and corticosteroids were prescribed according to local protocol. QuantiFERON TB Plus test (QFT-Plus; QuantiFERON@-TB Gold Plus, Qiagen, Germany) was undetermined (TB1: $0.08 \mathrm{IU} / \mathrm{ml}$; TB2 $0.26 \mathrm{IU} / \mathrm{ml}$; Mitogen: $0.02 \mathrm{IU} / \mathrm{ml}$ ).

Despite treatment, respiratory failure worsened: at day 4 from the admission arterial blood gas analysis performed with $\mathrm{FiO}_{2} 50 \%$ reported a $\mathrm{PaO} 2 / \mathrm{FiO}_{2}$ ratio of 101 . Due to this severe impairment of respiratory function, oxygen with a non-rebreather mask at $15 \mathrm{Lt} / \mathrm{min}$ was delivered. A chest
HRCT at day 6 from the admission to our Institute (day 13 from admission to Emergency Department) showed an increase in the number and extent of ground grass opacities with pattern "crazy paving" of the right lung (Fig. 1c). Due to the onset of a multi-organic failure, clinically represented by encephalopathy, diuresis contraction and hypotension, patient died at day 7 from referral to our Institute.

\section{Discussion}

Here, we describe a case of a patient with in vitro immune cell anergy affected by bilateral cavitary pulmonary TB and subsequent COVID-19-associated pneumonia with a worst outcome. The clinical spectrum of SARS-CoV-2 infection encompasses asymptomatic infection, mild upper respiratory tract illness and severe viral pneumonia with respiratory failure and even death. Initial signs and symptoms of this highly infectious disease are similar to other respiratory infections, such as TB [3], but clinical course and treatment differ among them with a higher mortality rate of COVID-19.

The pathogenicity of COVID-19 still remains unknown and experience with concomitant TB is extremely limited. It is well documented that certain viral infections, such as measles, have been known to exacerbate pulmonary TB, presumably as a result of depressed cellular immunity [4]. Influenza co-infection in TB cases is associated with a proinflammatory response, an increased mycobacterial load [5], and mortality rate in both animal models and patients $[6,7]$. Descriptive studies have reported a high prevalence of TB in cases of severe pandemic influenza, but available data about pandemic influenza and TB co-infection are low [7].

A recent study from China reported that persons with active or latent TB have increased susceptibility for SARSCoV-2 infection associated with rapid progression and severe involvement while a case of co-infection always from China reported with a good SARS-CoV-2 outcome [8]. Unfortunately, in our case, the patient died of COVID-19 
after 13 days from TB diagnosis. TB typically requires cellular immunity, in particular CD4-mediated immunity but not only as CD8 T cells play an important role $[9,10]$. TB disease may be transiently immunosuppressive. The combined effect of TB and SARS-COV-2 infection likely caused a pronounced lymphocytopenia [11] and consequently a CD4 + cell decrease as described in COVID-19 [12] and TB and SARS co-infection [13]. Lymphopenia is a reliable indicator of the severity and hospitalization of COVID-19 patients [11]. Various mechanisms of lymphopenia have been speculated, including lymphocyte death due to direct infection trough receptor ACE 2, direct damage of SARSCOV-2 to lymphatic organs and lymphocytes deficiency induced by pro-inflammatory cytokines, such as tumour necrosis factor (TNF) $\alpha$ and interleukin (IL)-6 [11, 14]. In our case, regarding TB-specific tests, the laboratory findings showed an indeterminate result of IGRA test. This result is important in light of the fact that in 2016, Auld et al. showed in large study that a negative or indeterminate score to IGRA in TB patients is associated with an increased risk of disseminated disease and death after initiating anti-tubercular treatment [15].

Our patient was immune-suppressed, as shown by the low lymphocyte number and by the lack of response to an immune-based assay likely by both disease, TB and COVID19 , and this may have contributed to the deterioration of the patient and death. IFN- $\gamma$ levels in QuantiFERON-tests correlate with lymphocyte count, and CD4 + cells play an important role against $M$. tuberculosis [16]. The response is mediated by $\mathrm{CD} 4$ or $\mathrm{CD} 8$ effector memory T cells. In COVID-19, the SARS-CoV-2-specific CD4 + T cells in blood were typically central memory, CD8 + T cells typically had a more effector phenotype [17]. The high M. tuberculosis load along with the infection due to SARS-CoV-2 may have paralyzed the immune system, as previously seen in patients with severe TB which may be the result of a compartmentalization of T-specific cells at the pathogen site [17]. In the pathogenesis of other infectious diseases, the elimination of effector T cells may occur when T cells encounter high concentrations of antigens [18, 19]. Moreover, in severe forms of TB, the high load of $M$. tuberculosis may generate inefficient dendritic cells functions by infecting newly recruited monocytes with the functional consequence of reducing the pool of specific IFNg-producing T cells [20].

From a clinical management prospective, in presence of a clinical suspect of COVID-19 in a patient with concurrent pulmonary $\mathrm{TB}$, it is important repeating the naso-pharingeal swab to provide an early diagnosis, promptly isolation precautions and specific treatment. Nevertheless, the bad prognosis of our patient confirms that in active $\mathrm{TB}$, a negative or indeterminate IGRA score can be interpreted as a prognostic marker of an immunodepressive status and a worst outcome in patients co-infected with SARS-CoV-2. In our case, COVID-19 was the precipitating factor of TB respiratory failure. The pathogenic hypothesis of this deterioration is unclear, a possible cause is the immunodepression of the patient with advanced TB along with the possible impairment of immune system due to SARS-CoV-2. In the present study, the indeterminate result of IGRA was a consequence of severe TB and likely, of the immunosuppressive effect of SARS-CoV-2 infection. We acknowledge that it may be unfeasible to perform in clinical setting routine IGRA to identify patients at risk for worst outcome, especially in poor countries.

We suggest to clinicians to be aware of the possibility of COVID-19-associated pneumonia in differential diagnosis of patients with active TB and new or worsening chest imaging during ongoing pandemic. This can be challenging because TB and COVID-19 share many similarities both in symptoms, as coughing, fever and dyspnea and in their radiological aspects, which can confound each other. In our opinion, COVID-19 can be a death risk factor in a frail population as TB patients, with a considerable impact on the healthcare system; further studies are urgent to optimize management of this co-infection.

Acknowledgements The authors thank all the nursing staff and sociohealth operators of Respiratory Infectious Diseases Unit, National Institute for Infectious Diseases "L. Spallanzani”, Rome. Our source of found is the Italian Ministry of Health, grant Ricerca Corrente, Research programme $n .4$ : tuberculosis.

Funding Our source of found is the Italian Ministry of Health, grant Ricerca Corrente, Research programme n.4: tuberculosis.

\section{Compliance with ethical standards}

Conflict of interests Patient was also included in a cohort of 69 patients described by Motta et al. [2]. The authors declare that they have no other competing interests.

Consent for publication Patient gave informed consent for publication

\section{References}

1. Dara M, Sotgiu G, Reichler MR, Chiang CY, Chee CBE, Migliori GB. New diseases and old threats: lessons from tuberculosis for the COVID-19 response. Int J Tuberc Lung Dis. 2020;24:544-5.

2. Motta I, Centis R, D'Ambrosio L, Garcia-Garcia J, Goletti D, Gualano G, et al. Tuberculosis, COVID-19 and migrants: preliminary analysis of deaths occurring in 69 patients from two cohorts. Pulmonology. 2020. https://doi.org/10.1016/j.pulmoe.2020.05.002 (Online ahead of print).

3. Ong CWM, Goletti D. Impact of the global COVID-19 outbreak on the management of other communicable diseases. Int $\mathrm{J}$ Tuberc Lung Dis. 2020;2:547-8.

4. Low JG, Lee CC, Leo YS, Low JG, Lee CC, Leo YS. Severe acute respiratory syndrome and pulmonary tuberculosis. Clin Infect Dis. 2004;38:e123-5. 
5. Mendy J, Jarju S, Heslop R, Bojang AL, Kampmann B, Sutherland JS. Changes in mycobacterium tuberculosis-specific immunity with influenza co-infection at time of TB diagnosis. Front Immunol. 2019;4:3093.

6. Redford PS, Mayer-Barber KD, McNab FW, Stavropoulos E, Wack A, Sher A, et al. Influenza A virus impairs control of Mycobacterium tuberculosis coinfection through a type I interferon receptor-dependent pathway. J Infect Dis. 2014;209:270-4.

7. Walaza S, Cohen C, Tempia S, Moyes J, Nguweneza A, Madhi $\mathrm{SA}$, et al. Influenza and tuberculosis co-infection: a systematic review. Influen Other Respir Viruses. 2020;14:77-91.

8. Chen Y, Wang Y, Fleming J, Yu Y, Gu Y, Yu Y, et al. Active or latent tuberculosis increases susceptibility to COVID19 and disease severity. MedRxiv. 2020. https://doi. org/10.1101/2020.03.10.20033795.

9. Amelio P, Portevin D, Hella J, Reither K, Kamwela L, Lweno O, et al. HIV infection functionally impairs mycobacterium tuberculosis-specific CD4 and CD8 T-Cell responses. J Virol. 2009;93:5.

10. Esmail H, Riou C, Bruyn ED, Lai RP, Harley YXR, Meintjes G, et al. The immune response to mycobacterium tuberculosis in HIV-1-coinfected persons. Annu Rev Immunol. 2018;36:603-38.

11. Tan L, Wang Q, Zhang D, Ding J, Huang Q, Tang YQ, et al. Lymphopenia predicts disease severity of COVID-19: a descriptive and predictive study. Signal Transduct Target Ther. 2020;5:33.

12. Weiskopf D, Schmitz K, Raadsen MP, Grifoni A, Okba NMA, Hendeman H, et al. Phenotype of SARS-CoV-2-specific T-cells in COVID-19 patients with acute respiratory distress syndrome. MedRxiv. 2020. https://doi.org/10.1101/2020.04.11.20062349.
13. Liu W, Fontanet A, Zhang PH, Zhan L, Xin ZT, Tang F, et al. Emerg. Infect. Dis. 2006;12:707-9.

14. Liao YC, Liang WG, Chen FW, Hsu JH, Yang JJ, Chang MS. IL-19 induces production of IL-6 and TNF-alpha and results in cell apoptosis through TNF-alpha. J Immunol. 2002;169:4288-97.

15. Auld SC, Lee SH, Click ES, Miramontes R, Day CL, Ghandi $\mathrm{NR}$, et al. IFN- $\gamma$ release assay result is associated with disease site and death in active tuberculosis. Ann Am ThoracSoc. 2016;13:2151-8.

16. Boyer-Suavet S, Cremoni M, Dupeyrat T, Zorzi K, Brglez V, Benzaken $\mathrm{S}$, et al. Functional immune assay using interferon-gamma could predict infectious events in end-stage kidney disease. Clinica Chimica Acta. 2020;502:287-92.

17. Petruccioli E, Petrone L, Vanini V, Sampaolesi A, Gualano G, et al. IFN $\gamma / \mathrm{TNF} \alpha$ specific-cells and effector memory phenotype associate with active tuberculosis. J Infect. 2013;66:475-86.

18. Moskophidis D, Lechner F, Pircher H, Zinkernagel R. Virus persistence in acutely infected immunocompetent mice by exhaustion of antiviral cytotoxic effector T cells. Nature. 1993;62:758-61.

19. Garcia S, DiSanto J, Stockinger B. Following the development of a CD4 T cell response in vivo: from activation to memory formation. Immunity. 1999;11:163-71.

20. Mariotti S, Teloni R, Iona E, Fattorini L, Giannoni F, Romagnoli $\mathrm{G}$, et al. Mycobacterium tuberculosis subverts the differentiation of human monocytes into dendritic cells. Eur J Immunol. 2002;32:3050-8. 